\title{
Urinary Neutrophil Gelatinase-Associated Lipocalin (uNGAL) in High Altitude: A Case Study
}

\author{
Scalzotto $E^{1}$, Corradi $V^{1}$, Brendolan $A^{1}$, Orrasch $M^{2}$, Fortunato $A^{3}$, Giavarina $D^{3}$ and Ronco $C^{1^{*}}$ \\ ${ }^{1}$ Department Nephrology Dialysis \& Transplantation, International Renal Research Institute (IRRIV), San Bortolo Hospital, Italy \\ ${ }^{2}$ Department Internal Medicine, Diabetes Center, Santa Chiara Hospital, Italy \\ ${ }^{3}$ Clinical Chemistry and Haematology Laboratory, San Bortolo Hospital, Italy
}

*Corresponding author: Claudio Ronco, Director of Department Nephrology Dialysis \& Transplantation International Renal Research Institute (IRRIV), San Bortolo Hospital VialeRodolfi, 37, 36100 Vicenza, Italy, Tel: +39 0444 753869; Fax: +39 0444753949 ; E-mail: cronco@goldnet.it

Rec date: Apr 26, 2014, Acc date: May 23, 2014, Pub date: May 27, 2014

Copyright: (c) 2014 Scalzotto E, et al. This is an open-access article distributed under the terms of the Creative Commons Attribution License, which permits unrestricted use, distribution, and reproduction in any medium, provided the original author and source are credited.

\begin{abstract}
Introduction and Aims: Medical problems occur at high altitude. The classical physiological responses to high altitude include hyperventilation, polycythemia and hypoxiemia. Type 1 diabetes (T1D) can participate in extreme altitude mountaineering. The prevalence of acute kidney injury (AKI) at high altitude is not known. We studied the renal function, by urinary Neutrophil gelatinase-associated lipocalin (UNGAL) in high altitude, as a promising biomarker for early detection of AKI.
\end{abstract}

Methods: The 2012 ADIQ Expedition team included 4 male Caucasians participants (prt): two with T1D and two non-diabetics. Urine Glucose, Protein, Urobilinogen, pH, Specific Gravity, Blood, Ketones, Nitrite and Leucocytes were determinated on-site by Aution Sticks. The uCreatinine and uUrea were measured by F360 analyzer. uNGAL concentration was measured by the ARCHITECT urine NGAL assay. Microalbumin concentration was obtained by Beckman Image. Urine Total Protein measured by Dimension Vista analyser. The data has been normalized for urinary creatinine.

Results: All expedition prt collected the urine samples $(5000,5600 \mathrm{~m})$. The urinary qualitative results by dipstick evaluation showed normal values in all cases. The values of glycaemia in T1D prt were high after arrival at basecamp (without presence of ketones). The UNGAL concentrations and UNGAL/uCrea ratios were also lower than 132 $\mathrm{ng} / \mathrm{ml}$ (cut-off of uNGAL) in all participants.

Conclusions: All the prt had normal UNGAL during the time of expedition, suggesting normal renal activity also confirmed by the analysis of the other parameters. The renal function in T1D prt was preserved despite the abnormal metabolic state represented by hyperglycemia. In this type of activity, level of training is especially important.

Keywords: NGAL (MeSH Terms); Diabetes mellitus, type 1 (MeSH Terms); Biological markers/urine (MeSH Terms); Glucose metabolism disorder (MeSH Terms); Extreme mountaineering; AKI

\section{Abbreviations:}

HAI: High Altitude Illness; AMS: Acute Mountain Sickness; HAPE: High Altitude Pulmonary Edema; HACE: High Altitude Cerebral Edema; AKI: Acute Kidney Injury; NGAL: Neutrophil GelatinaseAssociated Lipocalin

\section{Background}

Medical problems can occur at high altitude because of the low inspired which in turn is caused by the reduced barometric pressure. The classical physiological responses to high altitude include hyperventilation, polycythemia and hypoxemia [1]. High altitude illness (HAI) is the collective term for the unique cerebral and pulmonary syndromes that can occur following an initial ascent to high altitude or following a further ascent while already at high altitude. HAI includes acute mountain sickness (AMS) and high altitude cerebral edema (HACE), which afflicts the brain, and high altitude pulmonary edema (HAPE), which afflicts the lungs. They are induced by the hypoxic stress of high altitude and are characterized by extravascular fluid accumulation in the brain (AMS and HACE) and lungs (HAPE). AMS is clinically diagnosed based upon the appearance of typical symptoms in a person who lives at low altitude, but has recently ascended to high altitude (generally over $2000 \mathrm{~m}$ ). It is the most common form of high altitude illness and may occur following rapid ascent [2].

The kidneys play a crucial role in acclimatization and in AMS syndromes through their roles in regulating body fluids, electrolytes and acid-base homeostasis.

Extreme altitude mountaineering, which is defined as climbing to altitudes greater than $5000 \mathrm{~m}$, can create physiological demands, especially in type 1 diabetes (T1D) subjects. It is possible that these subjects might experience impaired pulmonary function [3]. As described by literature, type 1 diabetics can participate in extreme altitude mountaineering. However there are significant risks associated with such activity, including hypoglycemia, ketoacidosis, retinal haemorrhage [4] and proteinuria [5], with the additional difficulties in 
assessing glycemic control due to meter inaccuracy at high altitude [6] The prevalence of acute kidney injury (AKI) at high altitude is presently unknown, especially in T1D subjects.

Neutrophil Gelatinase-Associated Lipocalin (NGAL) is an irontransporting protein and it is produced and secreted by kidney tubule cells at low levels, but the amount produced and secreted into the urine and serum increases dramatically after ischemic, septic, or nephrotoxic injury of the kidneys. It is also upregulated in the lung during inflammation. NGAL rises rapidly in the nephron in response to a renal insult following Acute Kidney Injury (AKI) well before creatinine has risen. It has been put forward as an early, sensitive, and non-invasive biomarker for AKI. NGAL is also know to rise in conditions associated with oxidative stress, and oxidative stress has been implicated in AMS $[7,8]$. We decided to study the renal function, by urinary NGAL (UNGAL) -a promising biomarker for early detection of AKI- at high altitudes. The uNGAL seems to be more specific than plasma NGAL in assessing early kidney injury. Mishra et al. demonstrated that plasma NGAL had a good but lower performance than uNGAL for the diagnosis of AKI [9].

\section{Methods}

The 2012 Alpinisti Diabetici in Quota (ADIQ) Expedition team included 4 male Caucasians individuals: two with type 1 diabetes (T1D) [participants (prt) 1 e 2] and two non-diabetic [prt 3 and 4]. Prt 1- 35 years (yrs) and 4 yrs of T1D; Prt 2 - 42 yrs and 33yrs of T1D; Prt $3-55$ yrs; Prt $4-56$ yrs.

The four volunteers took part to the ADIQ expedition, and they were subjected to medical checks either before or after, according to behavioural regulations of such a kind of mountaineering expeditions. All volunteers collected their urinary samples and they gave them to our Laboratory, after having signed the informed consent. Because of that, it was not necessary to involve the Ethical Committee but all participants were informed about the responsibility that such an activity would require.

All expedition participants did not have kidney problems (the estimated glomerular filtration rate $>90 \mathrm{ml} / \mathrm{min} / 1,73 \mathrm{~m}^{2}$ ). The glomerular filtration rate before and after the expedition was estimate by the CKD-EPI equation [10].

The type 1 diabetic subjects were free of long-term diabetes complications and they had good metabolic control before the expedition $(\mathrm{HbA} 1 \mathrm{c}<7.5 \%)$.

All expedition participants collected urine samples every day after their arrival at the base-camp $(5000 \mathrm{~m})$. The subjects with T1D regulated glycaemia (CONTOUR * LINK, Bayer) every two hours (6-10 glycemic controls per day) to adjust the insulin treatment. The urine samples were maintained at $-20^{\circ} \mathrm{C}$ in special containers for cryopreservation (NALGENE ${ }^{m \text { }}$ ) and all the measurements were done in the same time, minimizing the laboratory variability to increase the inter assay precision.

The 2012 ADIQ Expedition to reach the summit of Sisha Pangma $(8027 \mathrm{~m})$ took place from 8 April to 11 May 2012 and was characterized by some intermediate stages: Kathmandu (1485 m), trekking to reach Base Camp, Base Camp (5000 m), Advanced Base Camp $(5600 \mathrm{~m})$, Camp $1(6400 \mathrm{~m})$ and Camp $2(6800 \mathrm{~m})$. Shisha Pangma, belonging to the Himalaya chain, is located in south-central Tibet, five kilometers from the border with Nepal and it is the fourteenth-highest mountain in the world.
Urine Glucose, Protein, Urobilinogen, pH, Specific Gravity, Blood, Ketones, Nitrite and Leucocytes were determinated on-site by using urine dipsticks and visual interpretation (Aution Sticks, A. Menarini diagnostics). Blood pressure (BP, systolic and diastolic) was determined by measuring device (Laica BM 1001); heart rate (HR) and $\mathrm{SO}_{2}$ were determined on-site by an oximeter (MIR, Spirotel).

In addition after return from the expedition we completed the measurements in laboratory. The uCreatinine and uUrea were measured by enzymatic method and urease kinetic respectively by F360 analyzer (Menarini diagnostic). uNGAL concentration was measured by the ARCHITECT urine NGAL assay (Abbott Laboratories). The cut-off of urinary NGAL is $132 \mathrm{ng} / \mathrm{ml}[11,12]$. Microalbumin concentration (MA; standard reference range: 0.2-4 $\mathrm{mg} / \mathrm{dL}$ ) was obtained by nephelometric method (Beckman Image). To confirm the data of protein measured in loco by the Aution stick, we measured urine total protein (uTP); standard reference range: 0-11.9 $\mathrm{mg} / \mathrm{dL}$ ) by pyrogallol red colorimetric method by Dimension Vista analyzer (SIEMENS) and the data has been normalized for urinary creatinine (uTP/uCrea;). Also, the uNGAL data has been normalized for urinary creatinine (uNGAL/uCrea).

AMS score was recorded during base camp period according to the Lake Louise Score (LLS); the participants filled out the questionnaire on their own [13]. The LLS allocates a score of 0 to 3 (symptom not present to severe) for symptoms of AMS (fatigue/weakness, dizzy/ light-headedness, and difficulty sleeping). A score of 3 or more in the presence of headache is consistent with AMS, a score of 6 or more with severe AMS.

\section{Results}

The main heights reached from the participants of 2012 ADIQ Expedition in the attempt to reach the summit of SishaPangma (8027 m) from 8 April to 11 May 2012 were $5000 \mathrm{~m}, 5600 \mathrm{~m}, 6400 \mathrm{~m}$ and $7000 \mathrm{~m}$. Due to the adverse weather conditions the 4 climbers males were not able to reach the summit, despite numerous attempts (temperature, wind, snow).

The values of glycaemia in T1D participants were high after arrival at base-camp (without presence of ketones).

All expedition participants collected the urine samples at $5000 \mathrm{~m}$ and at $5600 \mathrm{~m}$. The urinary volume in all participants was normal. The urinary qualitative results by dipstick evaluation showed normal values (Urobilinogen, pH, Specific Gravity, Blood, Ketones, Nitrite and Leucocytes) in all cases.

The urinary protein results obtained by dipstick evaluation and uTP laboratory measurement in all participants showed sign of no significant proteinuria (lower than $30 \mathrm{mg} / \mathrm{dl}$ ) only during the attempt to rich the summit. In any case all values of uTP/uCrea, and MA/ uCrea were normal (lower than their cut-off) in all participants over the time of the expedition.

The uNGAL concentrations and uNGAL/uCrea ratios were also lower than $132 \mathrm{ng} / \mathrm{ml}$ (cut-off) in all participants. According to their LLS at base camp altitude $(5000 \mathrm{~m})$ : Pt 1 and 3 showed severe AMS (LLS score 6 or more), while Pt 2 and Pt 4 did not show AMS (LLS of 0 to 3 ).

The trend of uNGAL/uCrea for all the participants is showed in Figure 1; the profiles of $\mathrm{BP}, \mathrm{HR}, \mathrm{SO}_{2}$, proteinuria and $\mathrm{uNGAL} / \mathrm{uCrea}$ for each participant over time and altitude are outlined in Figure 2 to 
5. In particular, participant 1 showed worse trend of $\mathrm{SO}_{2}$ (lower than $80 \%)$ compare with the other participants to the expedition.

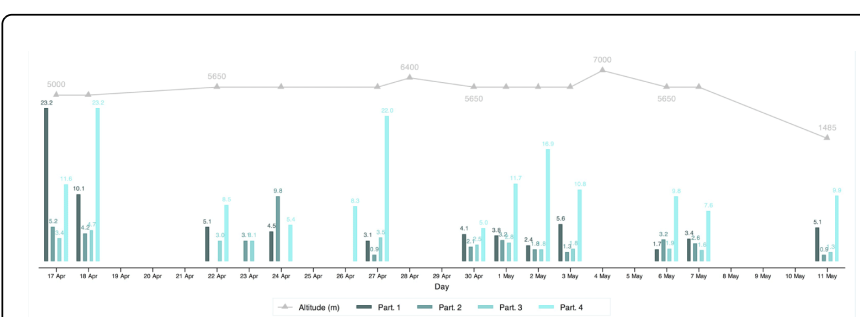

Figure 1: Trend of uNGAL/uCrea (y) by participant (different colour), time (x) and altitude

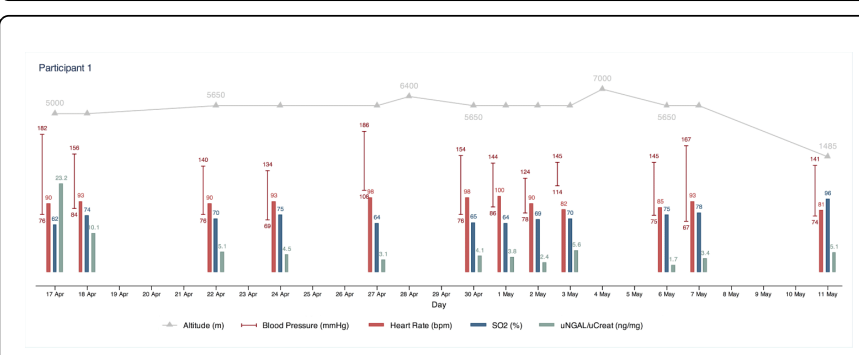

Figure 2: Profile of BP, HR, proteinuria, and uNGAL/uCrea (different colour) by time $(\mathrm{x})$ and altitude for participant 1

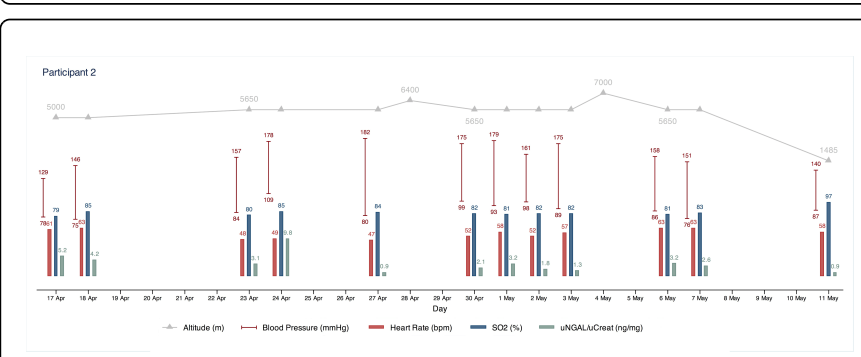

Figure 3: Profile of BP, HR, proteinuria, and uNGAL/uCrea (different colour) by time (x) and altitude for participant 2

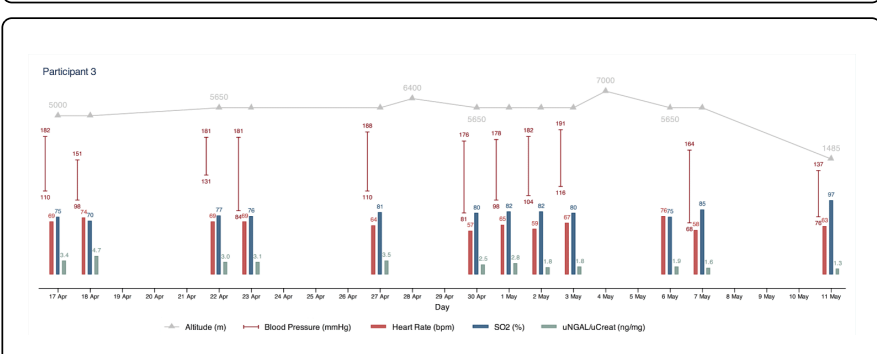

Figure 4: Profile of BP, HR, proteinuria, and uNGAL/uCrea (different colour) by time $(\mathrm{x})$ and altitude for participant 3

\section{Discussion}

This is the first study investigating a urinary marker to evaluate the kidney damage in high altitude in T1D subjects. Recently several reports showed controversial results about the tubular markers, including uNGAL, and the kidney disease progression in diabetic patients. The study from T1D indicated that urine NGAL level correlated with albumin/creatinine. T1D patients with higher albuminuria had higher uNGAL levels, which suggested that elevated uNGAL values might indicate kidney damage [14].

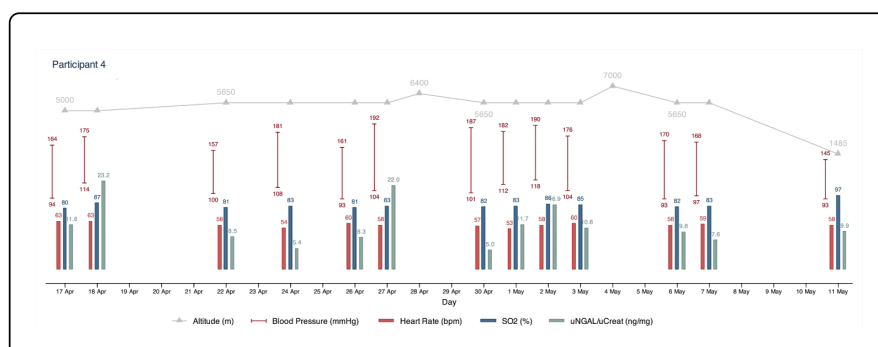

Figure 5: Profile of BP, HR, proteinuria, and uNGAL/uCrea (different colour) by time (x) and altitude for participant 4

In our study all participants showed a normal uNGAL/uCrea level during the time of the expedition, suggesting normal tubular renal activity. Moreover the absence of any significant proteinuria suggests a normal metabolic response at the high altitudes without evidence of glomerular damage.

The participant 1 showed worts trend of $\mathrm{SO}_{2}$ (lower than $80 \%$ ) compare with the other participants to the expedition, but the renal function was preserved despite the hyperglycaemia and hypoxia that usually play a critical role in the patogenesis of both AKI and CKD [15]. Probably, the hypoxia of participants 1 was due to hyperventilation (described into the questionnaire of LLS score) and difficult adaptation at high altitude.

The renal function in T1D participants was preserved despite the hyperglycaemia. In added none of the T1D subjects developed fresh retinal haemorrhages. Consistent with the changes observed in normal individuals, two of the major expeditions involving individuals with type 1 diabetes also report decreased glycaemic control, despite careful monitoring and appropriate changes in insulin dosing $[16,17]$.

The training level is particularly important in this type of activity but despite that, the climbers could also present problems due to environmental local conditions.

At very high altitude each individual is at risk for developing AMS, which, in its early stages, is characterized by headache, depressed appetite, gastrointestinal symptoms, fatigue and sleeping problems. AMS is accompanied by an increased sympathetic activity and an increase in plasma catecholamines concentration that could result in a deterioration of the glycemic control. It is difficult to predict the individual insulin requirements and the glucose profiles in response to exercise at very high altitude in subjects with type 1 diabetes. In addition, AMS may deteriorate glycemic control. These factors could therefore compromise safe trekking at very high altitude.

AMS score was self- recorded during base camp period by each of the participants, according to the Lake Louise Score questionnaire. A diagnosis of AMS is based on the presence of headache, at least one other symptom, and the total score of $\geq 4$.

Despite the presence of AMS in two subjects, the absence of AKI (by urinary markers results) suggests that the AMS and uNGAL may not be related. In any case, our results showed the absence of AKI during whole expedition. 
This study has some limitations. The poor number of the participants and the inability of collection of blood sample. It's not common that type 1 diabetics participate at the extreme mountaineering activity. However, our T1D participants are free from complications. Furthermore, at extreme altitude, highly motivated trekkers with type 1 diabetes but free from long-term complications present metabolic and cardiovascular parameters comparable with those of control subjects despite a worsening in metabolic control. This type of physical activity must be accompanied by careful glucose monitoring [17].

All our T1D participants regulated their own glucose levels and, in principle, determined their own insulin doses. Daily glucose levels were recorded using a handheld blood glucometers as usually. There were no problems with glucometers, the devices were protected with appropriate bags and carried next to the skin. Not rarely might happen that the insulin freezes because of the low temperatures. The collection of blood samples and the NGAL measurement on site in high altitude $(>5000 \mathrm{~m})$ was not possible, due to local conditions (altitude, temperature, sampling, handling and storage) and to the nonavailability of an assigned and qualified person. Moreover, it would have been necessary to use a point of care system for the quantitative determination of plasma NGAL. The performance of point of care can be affected by environmental stress like temperature, humidity, vibration, shock and altitude. This could lead to obtain erroneous measurement. In fact, falsely elevated or lowered readings may be attributed to changes in reagent enzymatic activities and kinetics [18-20].

Our study investigates the urinary markers of kidney function with a recommend procedure and following the guidelines. In particular, the collection and storage (in appropriate condition) of urine sample at high altitude is easy. This fact allows measuring all the parameters after the expedition in the same time by the same appropriate instruments, in controlled environmental conditions to improve the quality of the results.

\section{Conclusion}

Our data shows that UNGAL concentration did not rise in response at the high altitude condition. All the participants had normal uNGAL during the time of expedition, suggesting normal renal activity. The preservation of renal functions was also confirmed by the analysis of the other parameters.

The renal function in T1D participants was preserved despite the abnormal metabolic state represented by hyperglycemia (contrary to what occurs during extreme activity at sea level). In this type of activity, level of training is especially important. Moreover, all diabetic subjects have dealt with this activity were extremely trained to handle glucose monitoring and to vary dietary and insulin needs accordingly.

\section{Acknowledgements}

All participants in the studies are acknowledged for their support. The authors would like to thank A.D.I.Q (AlpinistiDiabetici in Quota) and its chairman, Dr. Marco Peruffo. Professor Claudio Ronco is the guarantor who takes full responsibility for the work as a whole, including the study design, access to data, and the decision to submit and publish the manuscript).
Prof.Claudio Ronco is the guarantor of this work and had full access to all the data in the study andtakes responsibility for the integrity of the data and the accuracy of the data analysis.

\section{Practical implications}

The practical implications of our study are summarized below:

1. The training kind and level are important for a good glycemic control in this extreme activity.

2. The controlled feeding according to every single subject metabolic necessities allowed to have a glycemic control through the diet.

3. The possibility of controlling urinary markers not only during the expedition, but also before and after, allowed having a better check of the possible kidney damage. This event is not rare in such a kind of extreme activities.

For these reasons we believe that our study might strengthen the extreme activity in high altitude of diabetics type 1 patients, with the necessary precautions.

\section{References}

1. West JB (2012) High-altitude medicine. Am J RespirCrit Care Med 186: 1229-1237.

2. Basnyat B, Murdoch DR (2003) High-altitude illness. Lancet 361: 1967-1974.

3. Strojek K, Ziora D, Sroczynski JW, Oklek K (1992) Pulmonary complications of type 1 (insulin-dependent) diabetic patients. Diabetologia 35: 1173-1176.

4. Moore K, Vizzard N, Coleman C, McMahon J, Hayes R, et al. (2001) Extreme altitude mountaineering and Type 1 diabetes; the Diabetes Federation of Ireland Kilimanjaro Expedition. Diabet Med 18: 749-755.

5. Mogensen CE, Vittinghus E (1975) Urinary albumin excretion during exercise in juvenile diabetes. A provocation test for early abnormalities. Scand J Clin Lab Invest 35: 295-300.

6. de Mol P, de Vries ST, de Koning EJ, Gans RO, Tack CJ, et al. (2011) Increased insulin requirements during exercise at very high altitude in type 1 diabetes. Diabetes Care 34: 591-595.

7. Bailey DM, Davies B, Young IS, Hullin DA, Seddon PS (2001) A potential role for free radical-mediated skeletal muscle soreness in the pathophysiology of acute mountain sickness. Aviat Space Environ Med 72: 513-521.

8. Bailey DM, Evans KA, James PE, McEneny J, Young IS, et al. (2009) Altered free radical metabolism in acute mountain sickness: implications for dynamic cerebral autoregulation and blood-brain barrier function. J Physiol 587: 73-85.

9. Mishra J, Dent C, Tarabishi R, Mitsnefes MM, Ma Q, et al. (2005) Neutrophil gelatinase-associated lipocalin (NGAL) as a biomarker for acute renal injury after cardiac surgery. Lancet 365: 1231-1238.

10. Levey AS, Stevens LA, Schmid CH, Zhang YL, Castro AF 3rd, et al. (2009) A new equation to estimate glomerular filtration rate. Ann Intern Med 150: 604-612.

11. (2009) Urine NGAL Product insert. Abbott Diagnostics Division, Longford, Ireland.

12. Clerico A, Galli C, Fortunato A, Ronco C (2012) Neutrophil gelatinaseassociated lipocalin (NGAL) as biomarker of acute kidney injury: a review of the laboratory characteristics and clinical evidences. ClinChem Lab Med 50: 1505-1517.

13. Hackett PH, Oelz O (1992) The Lake Louise consensus on the quantification of altitude illness. In. Burlington, VT: Queen City Printers: 327-330. 
Citation: Scalzotto E, Corradi V, Brendolan A, Orrasch M, Fortunato A, et al. (2014) Urinary Neutrophil Gelatinase-Associated Lipocalin (uNGAL) in High Altitude: A Case Study. J Diabetes Metab 5: 374. doi:10.4172/2155-6156.1000374

Page 5 of 5

14. Otu HH, Can H, Spentzos D, Nelson RG, Hanson RL, et al. (2007) Prediction of diabetic nephropathy using urine proteomic profiling 10 years prior to development of nephropathy. Diabetes Care 30: 638-643.

15. Evans RG, Gardiner BS, Smith DW, O'Connor PM (2008) Intrarenal oxygenation: unique challenges and the biophysical basis of homeostasis. Am J Physiol Renal Physiol 295: F1259-1270.

16. Pavan P, Sarto P, Merlo L, Casara D, Ponchia A, et al. (2003) Extreme altitude mountaineering and type 1 diabetes: the Cho Oyualpinisti in Alta Quota expedition. Diabetes Care 26: 3196-3197.

17. Pavan P, Sarto P, Merlo L, Casara D, Ponchia A, et al. (2004) Metabolic and cardiovascular parameters in type 1 diabetes at extreme altitude. Med Sci Sports Exerc 36: 1283-1289.
18. O'Malley JJ, Ulmer RW (1973) Thermal stability of glucose oxidase and its admixtures with synthetic polymers. BiotechnolBioeng 15: 917-925.

19. Gouda MD, Singh SA, Rao AG, Thakur MS, Karanth NG (2003) Thermal inactivation of glucose oxidase. Mechanism and stabilization using additives. J BiolChem 278: 24324-24333.

20. Louie RF, Ferguson WJ, Curtis CM, Vy JH, Kost GJ (2014) Vulnerability of point-of-care test reagents and instruments to environmental stresses: implications for health professionals and developers. ClinChem Lab Med 52: 325-335. 